\title{
La Flore Ptéridologique de Quelques Endroits Humides de la Région de Kédougou (Sénégal)
}

\section{Mingou Paterne}

Laboratoire de Botanique, Département de Botanique et Géologie, IFAN Ch. A. Diop, BP 206, Dakar

\section{Gueye Mathieu}

Laboratoire de Botanique, Département de Botanique et Géologie, UMI

3189, Environnement, Santé et Société, IFAN Ch. A. Diop, BP 206, Dakar

doi: 10.19044/esj.2017.v13n12p127 URL:http://dx.doi.org/10.19044/esj.2017.v13n12p127

\begin{abstract}
The study of the Pteridophyte flora of some wetlands in the Kedougou region of the Senegal Republic is the subject of this study. This analysis is based on the herbarium material of the pteridological flora harvested in this region and preserved in the herbaria of various institutions of IFAN, Dakar and Paris (P) also on the bibliographic data. This leads to a field mission to re-evaluate pteridophyte flora in this region. The results obtained show that the diversity of pteridophyte in the region of Kedougou is 11 species except for the very young species whose identification could not be made. These species grouped into 7 genera belong to 5 families of which the best represented are the Pteridaceae with 4 species. All the harvested and identified species were encountered in the Herbarium visited. Thus, in order to facilitate access to their identification, it is necessary to propose the tool which means that the document conforms to the identification key on the paper and the vegetative characters have been favored. Most of the pteridophyte were harvested at the Dindefelo cascade with an abundance of the species Adiantum philippense L. which is a species with large distribution because it is found in almost all the prospected places. All of these species are restricted to wetlands and shaded areas with occupation of all habitats (terrestrial, aquatic, saxicolous and epiphytic).
\end{abstract}

Keywords: Pteridophyte, wetlands, Kedougou, habitat, diversity

\section{Résumé}

L'analyse de la flore des Ptéridophytes de quelques endroits humides de la région de Kédougou, en République du Sénégal fait l'objet de cette étude. Cette analyse est fondée à la fois sur le matériel d'herbier de la flore ptéridologique récolté dans cette région et conservé dans les herbiers de 
diverses institutions de l'IFAN, de Dakar et de Paris (P) mais aussi sur les données bibliographiques. Ce qui a conduit à faire une mission de terrain afin de réévaluer la ptéridoflore dans cette région. Les résultats obtenus montrent que la diversité des ptéridophytes dans la région de Kédougou est de 11 espèces à l'exception de l'espèce très jeune dont l'identification n'a pu être faite. Ces espèces regroupées en 7 genres appartiennent à 5 familles dont les mieux représentées sont les Pteridaceae avec 4 espèces. L'intégralité des espèces récoltées et identifiées ont été rencontrées dans les Herbiers visités. Ainsi, pour faciliter l'accès à leur identification il a paru nécessaire de proposer l'outil qui manquait jusqu'à présent c'est à dire une clé d'identification sur papier dont les caractères végétatifs ont été favorisé. La majorité des ptéridophytes ont été récolté au niveau de la cascade de Dindéfélo avec une abondance de l'espèce Adiantum philippense L. qui est une espèce à large distribution car retrouvée dans presque tous les endroits prospectées. L'ensemble de ces espèces sont cantonnées dans les milieux humides (cascades, berges d'eaux) et dans les zones ombragées avec une occupation de toutes les diverses habitats (terrestres, aquatiques, saxicoles et épiphytes).

Mots clés : Ptéridophytes, endroits humides, Kédougou, habitats, diversité

\section{Introduction}

Les Ptéridophytes sont des cryptogames vasculaires relativement bien représentées dans le Sud du Sénégal où elles ne constituent toutefois pas de populations distinctes (Bâ et Noba, 2001).

Les Ptéridophytes ou "fougères et alliées" étaient des termes traditionnellement utilisés pour regrouper les Lycophytes et les Monilophytes (=fougères sensu Pryer et al., 2001). Ces dernières comprennent environs 12.000 espèces existantes (Moran R.C., 2008) et sont les plus proches parents vivants des plantes à graines (Pryer et al., 2001 et 2004 ; Smith et al., 2006 ; Christenhusz et al., 2011). Les ptéridophytes occupent tous les milieux (aquatiques, terrestres et épiphytes) et sont rencontrées aussi bien que dans les zones arides que dans les zones humides. Mais elles sont plus communes des régions montagneuses humides et tempérées et dans les régions de plateau tropicales et subtropicales, spécialement dans les forêts fermées (David, 1993; Johns, 1985; Parris, 1985; Tryon, 1985).

La région de Kédougou présente de telles caractéristiques favorables au développement de la ptéridoflore par la présence de zones humides à savoir les cascades ou chutes d'eau, cours d'eau et des zones ombragées qui sont des milieux de prédilection des «fougères ». La ptéridoflore est très bien peu connue au Sénégal. En effet, Bâ et Noba (2001), signalaient déjà la 
rareté, l'ancienneté et l'insuffisance des informations disponibles sur les ptéridophytes au Sénégal. En plus, la région de Kédougou est connue pour ses intenses activités minières et ses attractions touristiques du fait de la présence du Parc Niokolo-Koba, la réserve des chimpanzés à Dindéfelo et de ses nombreuses cascades. Ces activités contribuent à la destruction des habitats entraînant une modification de la composition floristique d'où, l'urgence d'inventaires pour mieux connaître, sauvegarder et comme le suggèrent Bâ et Noba (2001) assurer la protection et la conservation des espèces sensibles. Au Sénégal, les Ptéridophytes ne seraient représentées que par 38 espèces (Bâ et Noba, 2001). Malheureusement, ceci est une estimation basée sur des données anciennes et fragmentaires car, aucun inventaire systématique des ptéridophytes n'est à ce jour effectué. Il faut cependant rappeler que ces plantes cryptogames ont pour la plupart, une grande importance dans le maintien et, le développement des écosystèmes (Aldasoro et al., 2004).

Le présent travail étudie la ptéridoflore de quelques endroits humides de la région de Kédougou pour une meilleure connaissance de ce groupe et se propose d'établir une clé d'identification de ces espèces et d'analyser leur distribution dans la région.

\section{Matériel et méthodes}

\section{Contexte général du pays}

Le Sénégal présente divers types de végétations (steppes, savanes, zones de cultures, mangroves et forêts) en relation avec le gradient climatique et les conditions topo-édaphiques.

C'est ainsi qu'on retrouve du Nord au Sud (figure 1) :

les steppes arbustive et arborée, situées dans le domaine sahélien, sont caractérisées par une strate herbacée plus ou moins continue et parsemée d'espèces ligneuses épineuses telles qu'Acacia tortilis subsp. raddiana, Acacia senegal, Acacia seyal et Balanites aegyptiaca;

les savanes arbustives et arborée ou boisées, situées vers le centre du pays, dans le domaine soudanien, sont caractérisées par une strate ligneuse comme Cordyla pinnata, Pterocarpus erinaceus, Parkia biglobosa, Detarium senegalense, Daniellia oliveri, etc. ;

les forêts claire, dense sèche, et galerie, situées dans le domaine guinéen, sont caractérisées par des espèces telles qu'Erythrophleum guineense, Detarium senegalense, Parinari excelsa, Raphia sudanica, Carapa procera, Elaeis guineensis, etc.

Ces principaux types de végétation (figure 1) occupent une superficie de 19679450 ha dont $44 \%$ de savane, $27 \%$ de zones de cultures, $18 \%$ de steppe, $4 \%$ de forêts et $1 \%$ de mangrove (MEPN, 2014). 


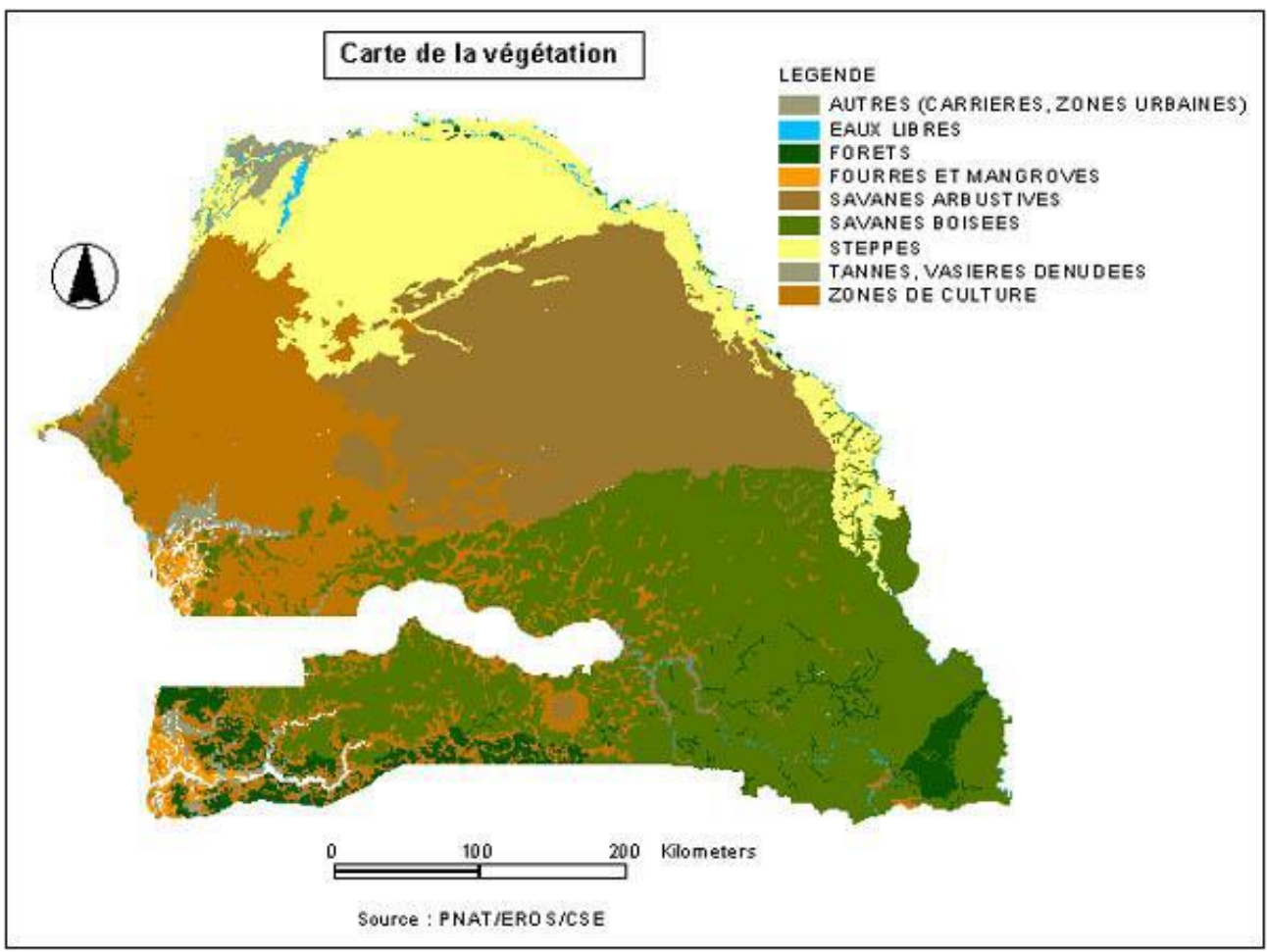

Figure 1. Principaux types de végétation du Sénégal (Source: MEPN, 2014)

\section{Présentation de la zone d'étude}

Située dans le Sénégal oriental, la région de Kédougou est limitée au sud par la République de Guinée, à l'est par le Mali, au nord et à l'ouest par la région de Tambacounda (Figure 2). Elle couvre une superficie de 16816 $\mathrm{Km}^{2}$ et compte trois départements (Kédougou, Saraya et Salémata). La région est caractérisée à la fois par un relief très accidenté avec des altitudes dépassant les $500 \mathrm{~m}$ et par un climat de type sub-guinéen avec une pluviométrie de plus de $1000 \mathrm{~mm} / \mathrm{an}$. Ce qui lui confère une végétation constituée de forêts sèches et par rapport aux conditions topo-édaphiques particulières on y rencontre des forêts galeries le long des cours d'eau. 


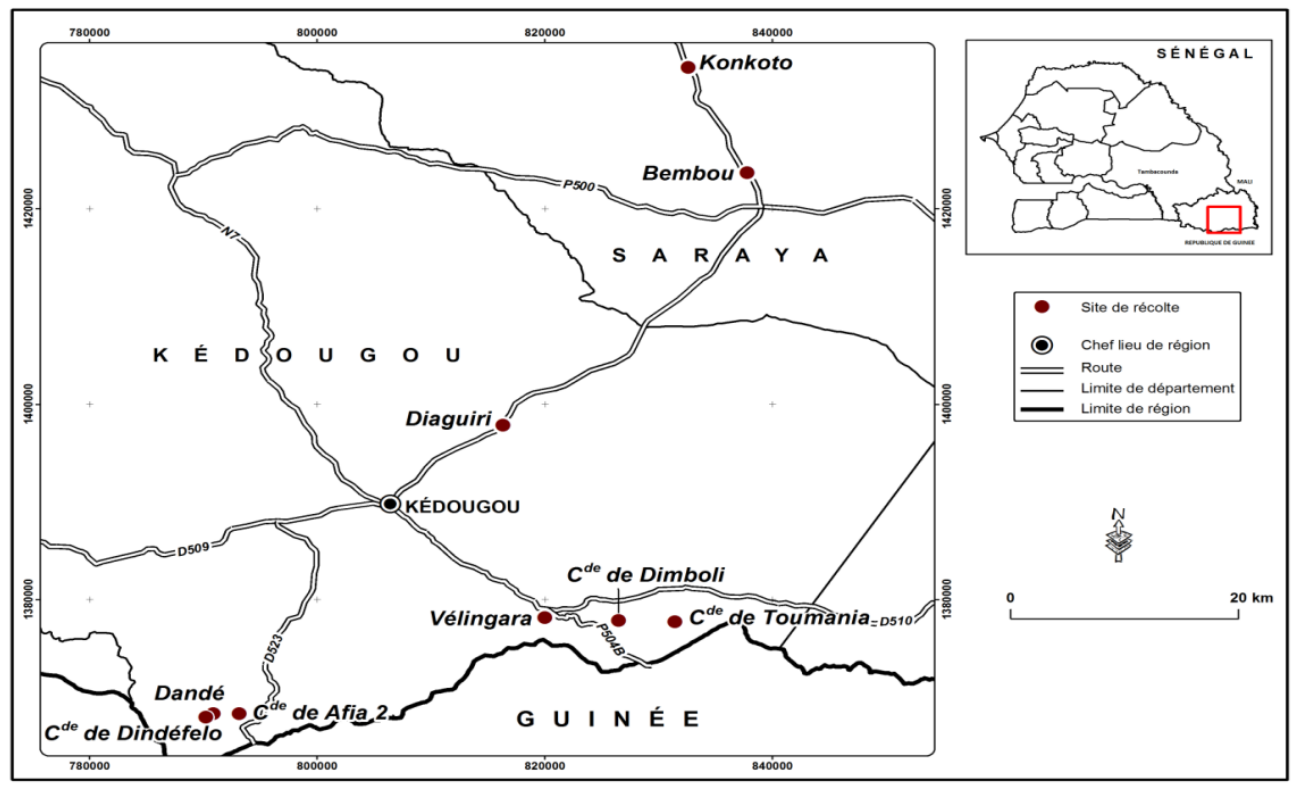

Figure 2. Carte des différentes Zones prospectées dans la région de Kédougou (Sénégal)

À Kédougou, les cascades prospectées sont celles de Dindéfélo (Dindéfélo, Afia 2), Dimboli, Dandé, Toumania ; les zones de Diaguiri, Bembou, Konkoto, et Vélingara ont aussi été prospectées (Figure 2). Les prospections ont ciblé particulièrement les zones humides à savoir les cours d'eaux, ruisseaux et rivières et des zones ombragées qui sont des lieux de prédilection des fougères. Les prospections ont été effectuées pendant la saison pluvieuse aux mois de juillet-août, mois les plus pluvieux au Sénégal.

\section{Récolte d'échantillons}

A la base une synthèse bibliographique a été faite en premier lieu afin de déterminer et de dresser une première liste des ptéridophytes du Sénégal. Les principaux documents consultés sont la flore du Sénégal (Berhaut, 1967), des flores africaines (Tardieu-Blot, 1953 et 1964 ; Roux, 2001 et 2003) et Bâ et Noba (2001). Les échantillons des herbiers de l'Institut Fondamental d'Afrique Noire (IFAN), du Département de Biologie Végétale de la Faculté des Sciences et Techniques de l'Université Cheikh Anta Diop de Dakar (DAKAR) et du Muséum National d'Histoire Naturelle (MNHN) de Paris $(\mathrm{P})$.

Une fois les données bibliographiques réunies, les sites à prospecter ont été sélectionnés et de nouvelles récoltes ont été faites afin de réévaluer la diversité des ptéridophytes dans la région de Kédougou. Ces récoltes n'ont porté que sur les fougères rencontrées sur les sites visités. La méthode d'échantillonnage subjectif a été retenue qui consiste à inventorier toutes les espèces de ptéridophytes sur une surface d'observation définie en fonction de 
l'hétérogéneité et la représentativité des milieux (zones humides, mares, rivières et cascades). Cette méthode nous a permis de parcourir parfois de longues distances par exemple nous avons prospecté du début de la piste (plus de $600 \mathrm{~m}$ de part et d'autre de celle-ci) de la cascade Dindéfélo jusqu'à la cascade elle même. Tous les ptéridophytes observées sont récoltées. Avant la récolte d'un échantillon, des informations précises correspondantes à cette espèce sont notées sur la fiche de collecte affectée à l'échantillon à savoir les coordonnées GPS, l'habitat, les dimensions de l'espèce (longueur et largeur de la fronde, longueur du pétiole...), la couleur des différentes parties (pétiole et limbe) et en plus des photos complètes de l'espèce sont prises dans son milieu. Celles-ci aideront plus tard à la description de l'espèce. Ensuite, à l'aide d'un sécateur ou d'un couteau l'échantillon est prélevé, s'il est fixé au sol on creuse au plus profond de la terre afin d'avoir l'intégrale des racines et le dérocher s'il est fixé sur un rocher. On récolte les échantillons de tel sorte à obtenir toutes les parties c'est-à-dire de la racine aux frondes. Les spécimens prélevés sont de préférence matures c'est-à-dire que les frondes doivent être fertiles avec des sporanges. À défaut d'un spécimen mature, un spécimen stérile est récolté. L'identification de l'espèce est parfois faite dans l'immédiat sur le terrain.

\section{Identification des récoltes}

Au laboratoire, l'étape du tri et de l'identification des spécimens a été procédée. L'identification est faite à l'aide de flores (Berhaut, 1967 ; Tardieu-Blot, 1953 et 1964 ; Roux, 2001 et 2003). Conjointement, dans le but d'identifier et/ou de confirmer les identifications, une confrontation des échantillons nouvellement collectés avec des échantillons d'herbiers a été faite. À défaut d'une identification sure, l'avis d'un spécialiste a été sollicité. Il convient de noter que la difficulté d'identification des taxons au rang spécifique est due au stade juvénile de certains individus au moment de la récolte. Les spécimens triés, séchés et identifiés sont montés puis conservés à l'herbier de l'IFAN. La nomenclature utilisée est celle de Smith et al. (2006) et de Schuettpelz et al. (2007) actualisée à quelques exceptions près par Christenhusz et al. (2011). Quant à la liste des synonymes nous avons consulté les bases Tropicos (BDD du Missouri Botanical Garden, http://www.tropicos.org); et The Plant list (http://www.theplantlist.org).

\section{Résultats}

Sur la base des données bibliographiques et des échantillons présentes dans les herbiers (IFAN, Dakar et Paris), des centaines d'échantillons récoltés et des bouleversements systématiques de la taxinomie (des familles dissoutes, des genres transférés et des noms d'espèces tombés en synonymie), la diversité des Ptéridophytes dans la région de Kédougou est 
de 11 espèces (Tableau 1 et Planche 1). Hormis une espèce dont le genre n'a pu être identifié, elles appartiennent à 7 genres distribués dans 5 familles dont les mieux représentées sont les Pteridaceae avec 4 espèces suivies des Dryopteridaceae et des Thelypteridaceae avec 2 espèces chacune. Toutes les espèces récoltées et clairement identifiées ont été rencontrées dans les Herbiers visités mais, la confusion demeure pour les récoltes et les parts d'herbiers qui n'ont pas été identifiés jusqu'au rang d'espèce et pour la récolte dont nous n'avons pas pu déterminer le genre. À noter, que la littérature ne signale et, ne donne aucune information sur la distribution régionale des espèces.

Tableau 1. Liste des Ptéridophytes de la région de Kédougou (Sénégal)

\begin{tabular}{|c|c|c|c|c|}
\hline Ordre & Famille & Espèce & Herbier & $\mathrm{N}^{\circ}$ Récolte \\
\hline & & Espèce sp. & $\begin{array}{l}\text { Paris, } \\
\text { Dakar, } \\
\text { IFAN }\end{array}$ & DF11, DF12 \\
\hline $\begin{array}{l}\text { Saviniales } \\
\text { Bartl }\end{array}$ & $\begin{array}{l}\text { Marsileaceae } \\
\text { Mirbel }\end{array}$ & Marsilea sp. & $\begin{array}{l}\text { Paris, } \\
\text { Dakar, } \\
\text { IFAN }\end{array}$ & DG06 \\
\hline \multirow{9}{*}{$\begin{array}{l}\text { Polypodiales } \\
\text { Link }\end{array}$} & \multirow{2}{*}{$\begin{array}{l}\text { Dryopteridaceae } \\
\text { Herter }\end{array}$} & $\begin{array}{l}\text { Bolbitis acrostichoides } \\
\text { (Afzel. Ex Sw.) Ching }\end{array}$ & $\begin{array}{l}\text { Paris, } \\
\text { Dakar, } \\
\text { IFAN }\end{array}$ & DF10 \\
\hline & & $\begin{array}{c}\text { Bolbitis heudolotii (Bory) } \\
\text { Alston }\end{array}$ & $\begin{array}{l}\text { Paris, } \\
\text { Dakar, } \\
\text { IFAN }\end{array}$ & DF4 \\
\hline & $\begin{array}{l}\text { Nephrolepidaceae } \\
\text { Pichi-Serm. }\end{array}$ & $\begin{array}{l}\text { Nephrolepis bisserata } \\
\text { (Sw.) Schott }\end{array}$ & $\begin{array}{l}\text { Paris, } \\
\text { Dakar, } \\
\text { IFAN }\end{array}$ & $\overline{D F} 15$ \\
\hline & \multirow{4}{*}{$\begin{array}{l}\text { Pteridaceae } \\
\text { E.D.M. Kirchn. }\end{array}$} & Adiantum philippense L. & $\begin{array}{l}\text { Paris, } \\
\text { Dakar, } \\
\text { IFAN }\end{array}$ & $\begin{array}{c}\text { DF1, DF2, DF7, } \\
\text { DF13, DF14, } \\
\text { DF16, DF17, } \\
\text { DB01, DB05, } \\
\text { TN01, DG02, } \\
\text { DG03, BM01, } \\
\text { KK01, KK02, } \\
\text { KK03, KB01, } \\
\text { KB03 }\end{array}$ \\
\hline & & $\begin{array}{c}\text { Adiantum schweinfurthii } \\
\text { Kuhn }\end{array}$ & $\begin{array}{l}\text { Paris, } \\
\text { Dakar, } \\
\text { IFAN }\end{array}$ & $\begin{array}{l}\text { DF5, DF6, AF1, } \\
\text { DD1, }\end{array}$ \\
\hline & & $\begin{array}{c}\text { Pellaea doniana J.Sm. ex } \\
\text { Hook. }\end{array}$ & $\begin{array}{l}\text { Paris, } \\
\text { Dakar, } \\
\text { IFAN }\end{array}$ & DF8, AF2, DD2, \\
\hline & & Pteris sp. & $\begin{array}{l}\text { Paris, } \\
\text { Dakar, } \\
\text { IFAN }\end{array}$ & DF9 \\
\hline & \multirow{2}{*}{$\begin{array}{l}\text { Thelypteridaceae } \\
\text { Ching ex Pichi- } \\
\text { sermoli }\end{array}$} & Cyclosorus sp. & $\begin{array}{l}\text { Paris, } \\
\text { Dakar, } \\
\text { IFAN }\end{array}$ & AF5 \\
\hline & & $\begin{array}{l}\text { Cyclosorus dentatus } \\
\text { (Forssk.) Ching }\end{array}$ & $\begin{array}{l}\text { Paris, } \\
\text { Dakar, } \\
\text { IFAN }\end{array}$ & DF3, DF18 \\
\hline
\end{tabular}




\section{Taxinomie et catalogue synonymique des taxons présents à Kédougou}

$\mathrm{Au}$ regard des connaissances parcellaires et anciennes sur ce groupe, ce catalogue synonymique des ptéridophytes inventoriés ci-dessous est basé sur les travaux de Smith et al. (2006) et de Schuettpelz et al. (2007) actualisés par Christenhusz et al. (2011). La liste des synonymes a été établi en se référant en plus à des bases de données comme Tropicos et The Plant list, régulièrement mises à jour.

Espèce sp. (Planche 1-A)

\section{Famille des Dryopteridaceae}

Bolbitis acrostichoides (Afzel. ex Sw.) Ching (Planche 1-B)

= Hemionitis acrostichoides Sw. = Polybotrya acrostichoides (Sw.) Kuhn = Leptochilus acrostichoides (Sw.) C. Chr. = Campium acrostichoides (Sw.) Copel.

Bolbitis heudolotii (Bory) Alston (Planche 1-C)

= Gymnopteris heudelotii Bory = Anapausia heudolotii (Bory) C. Presl = Acrostichum heudelotii (Bory) Hook. = Acrostichum heudelotii var. minor Hook. $=$ Chrysodium heudelotii (Bory) Kuhn $=$ Leptochilus heudelotii (Bory)

C. Chr. = Campium heudelotii (Bory) Copel.

\section{Famille des Marsileaceae}

Marsilea sp. (Planche 1-D)

\section{Famille des Nephrolepidaceae}

Nephrolepis bisserata (Sw.) Schott (Planche 1-E)

= Aspidium acuminatum Willd. = Aspidium biserratum $\mathrm{Sw}$.

\section{Famille des Pteridaceae}

Adiantum philippense L. (Planche 1-F)

= Adiantum lunulatum Burm. f. = Pteris lunulatum (Burm. f.) Retz. = Adiantum philippense var. lobatum C. Chr. = Adiantum lunulatum Cav. = *Adiantum arcuatum $\mathrm{Sw}$.

Adiantum schweinfurthii Kuhn (Planche 1-G)

= Adiantum chevalieri Christ

Pellaea doniana J.Sm. ex Hook. (Planche 1-H)

= Pteris dura Willd. = Pellaea dura (Willd.) Hook. = Pellaea holstii Hieron.

$=$ Pellaea schweinfurthii (Hieron.) Diels = Pteris burkeana Hook. = Pteridella dura Mett. ex Kuhn

Pteris sp. (Planche 1-I)

\section{Famille des Thelypteridaceae}

Cyclosorus sp. (Planche 1-J) 
Cyclosorus dentatus (Forssk.) Ching (Planche 1-K)

$=$ Christella dentata (Forssk.) Brownsey $\&$ Jermy $=$ Polypodium dentatum Forssk. $=$ Polypodium molle Jacq. $=$ Aspidium molle $\mathrm{Sw} .=$ Nephrodium molle (Sw.) R. Br. = Nephrodium parasiticum (L.) Desv. = Dryopteris gongylodes (Schkuhr) Link = Thelypteris dentata (Forssk.) E.St.John = Cyclosorus elatus vel sp. Aff.

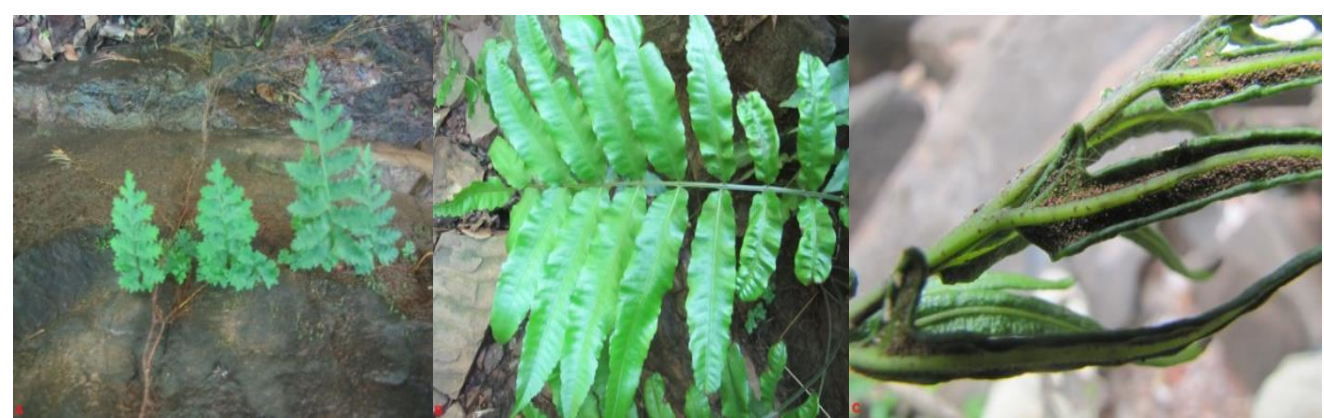

A

B

$\mathbf{C}$

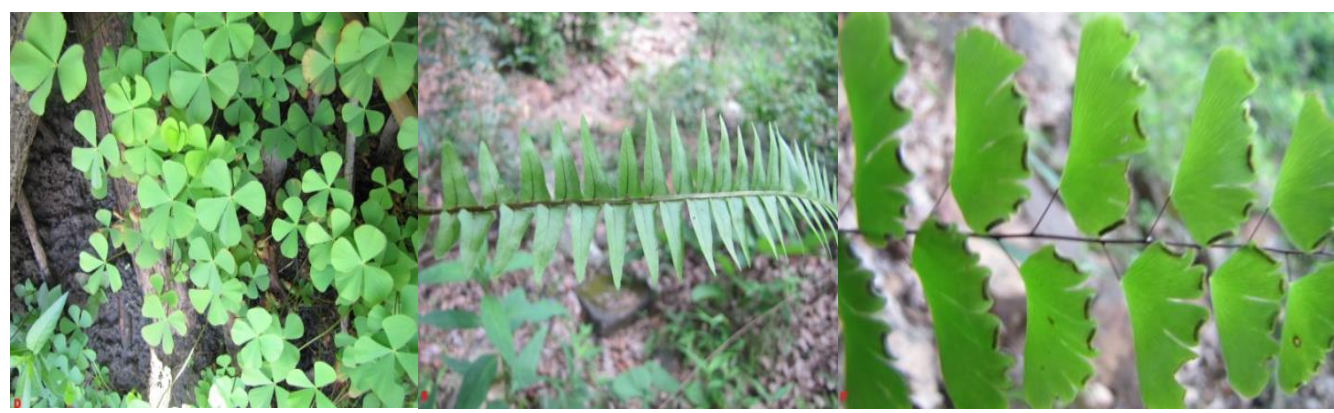

D

$\mathbf{E}$

$\mathbf{F}$

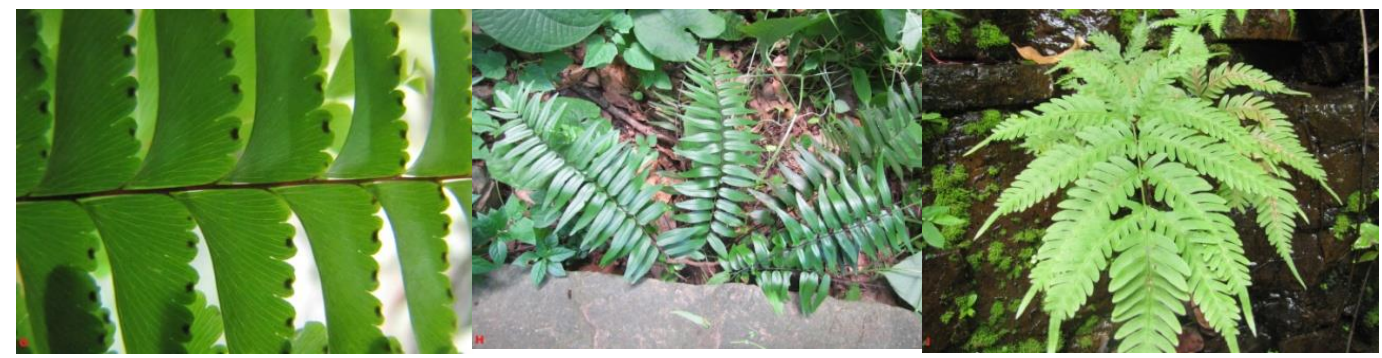

G

H

I 


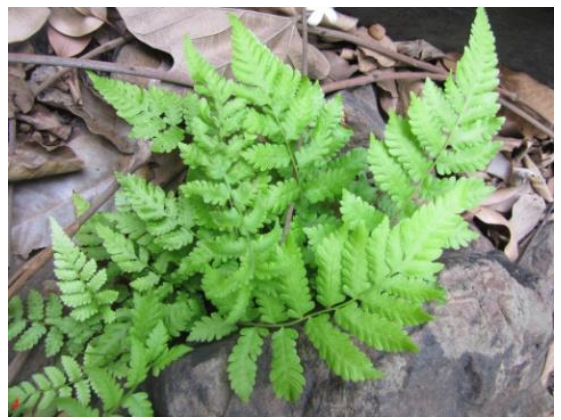

$\mathbf{J}$

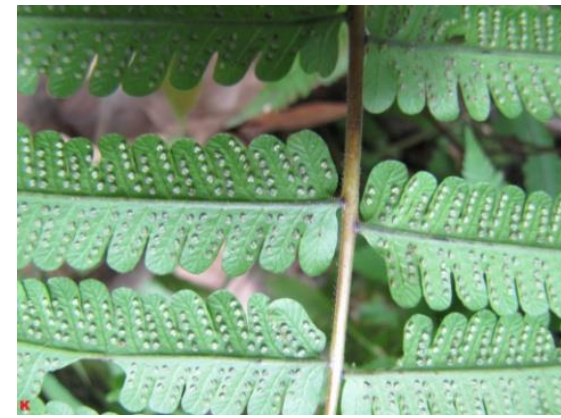

$\mathbf{K}$

Planche 1 : Quelques espèces représentatives des Ptéridophytes de la région de Kédougou in situ (Photos Bot_IFAN 2014). A. Espèce sp. B. Bolbitis acrostichoides (Afzel. ex Sw.).

C. Bolbitis heudolotii (Bory) Alston Ching. D. Marsilea sp.

E. Nephrolepis bisserata (Sw.) Schott. F. Adiantum philippense L.

G. Adiantum schweinfurthii Kuhn. H. Pellaea doniana J.Sm. ex Hook. I. Pteris sp. J. Cyclosorus sp.

K. Cyclosorus dentatus (Forssk.) Ching

\section{Clé d'identification des Ptéridophytes de la région de Kédougou} (Sénégal)

Cette clé dichotomique suivante débute par des caractères facilement observables. Ainsi, une priorité est donnée aux caractères végétatifs car, souvent un problème d'identification se pose au rang de l'espèce suivant que celle-ci n'est pas mature c'est-à-dire pas encore fertile (absence de sores). La clé proposée est adaptée aussi bien aux spécimens stériles qu'aux fertiles.

1. a/Fougères aquatiques ou subaquatiques vivant en eau douce ou dans la boue. .2

b/Fougères terrestres, lithophytes et/ou épiphytes....................3 2. a/Fougères aquatiques dans la boue ; frondes formées de 4 folioles deltoïde-cunéiforme; rhizome longuement rampant portant des poils Marsilea sp.

b/Fougères subaquatiques en eau douce; frondes pennées à pennes étroites, penne terminale lobée; nervures latérales très peu distinguées ; rhizome longuement rampant...............................Bolbitis heudelotii 3. a/Frondes bipennées-pinnatifides..............................4 b/Frondes simplement pennées..................................5

4. a/Frondes bipennées longues de 7 à plus de $40 \mathrm{~cm}$, pennes latérales courtement pétiolulées, première paire de pennes inférieures bipartite; petites écailles à la base du pétiole ; nervures libres; rhizome dressé, écailleux Pteris sp. 
b/Frondes bipennées-pinnatifides ; 2 grands faisceaux vasculaires à l'intérieur du pétiole; nervures et nervilles poilues; rhizome dressé ou rampant, écailleux

5. a/Frondes simplement pennées ; sores protégés par un repli du limbe, limbe généralement coriace, portant une nervation......................................................... 7

b/Frondes simplement pennées ; sores non protégés par un repli du limbe..... .8

6. a/Frondes touffues ; rhizome dressé, écailleux Cyclosorus sp.

b/Frondes rapprochées; pétiole canaliculé, écailleux à la base ; rhizome courtement rampant, écailleux. Cyclosorus dentatus 7. a/Frondes simplement pennées-pennatifides; pennes latérales alternes, peu nombreuses 6-20 paires, très longuement pétiolulées, semielliptiques, dimidiées; pétiole noir brillant non canaliculé, glabre..........................................Adiantum philippense

$\mathrm{b} /$ Frondes simplement pennées; pennes latérales alternes, très nombreuses 15-40 paires, courtement pétiolulées à sessiles, oblonguesrhomboïdales, dimidiées; pétiole noir brillant, canaliculé, écailleux à la base Adiantum schweinfurthii 8. a/Pennes latérales courtement à moyennement pétiolulées.

b/Pennes latérales sessiles, alternes, lancéolées à bases arrondies, subégales, marges entières ou légèrement serrulées, extrémité des pennes aiguës ; pétiole écailleux à la base. .Nephrolepis bisserata 9. a/Rhizome dressé, écailleux; 3-20 paires de pennes latérales, lancéolées, alternes, marges entières très peu effilées vers l'extrémité ; penne terminale semblable aux latérales; pétiole écailleux; rachis tomenteux. Pellaea doniana

b/Rhizome court rampant, 5-12 paires de pennes latérales, linéaireslancéolées, alternes, marges entières ou finement dentelées à \pm crénelées ; penne terminale semblable aux latérales, non lobée ; nervures latérales très distinguées; pétiole écailleux à la base, non canaliculé ; rachis glabre. Bolbitis acrostichoïdes

\section{Distribution des espèces}

Du point de vue de la diversité biologique, la région de Kédougou paraît favorisée par sa situation géographique. Ainsi, la cascade de Dindéfélo dispose de la plus grande diversité ptéridophytes avec 10 espèces [Adiantum philippense L., Adiantum schweinfurthii Kuhn (Figure 3), Bolbitis acrostichoides (Afzel. ex Sw.) Ching, Bolbitis heudelotii (Bory) Alston, espèce sp., Cyclosorus sp., Cyclosorus dentatus (Forssk.) Ching, Pteris sp., Pellaea doniana J.Sm. ex Hook. et Nephrolepis bisserata (Sw.) Schott 
(Figure 4)]. Ensuite, viennent les cascades de Dimboli, Toumania, Afia2 et Dandé qui sont aussi caractérisées par la présence des [A. philippense et $A$. schweinfurthii (Figure 3) mais aussi des espèces comme B. heudelotii, $C$. dentatus, Cyclosorus sp. (Figure 4)].

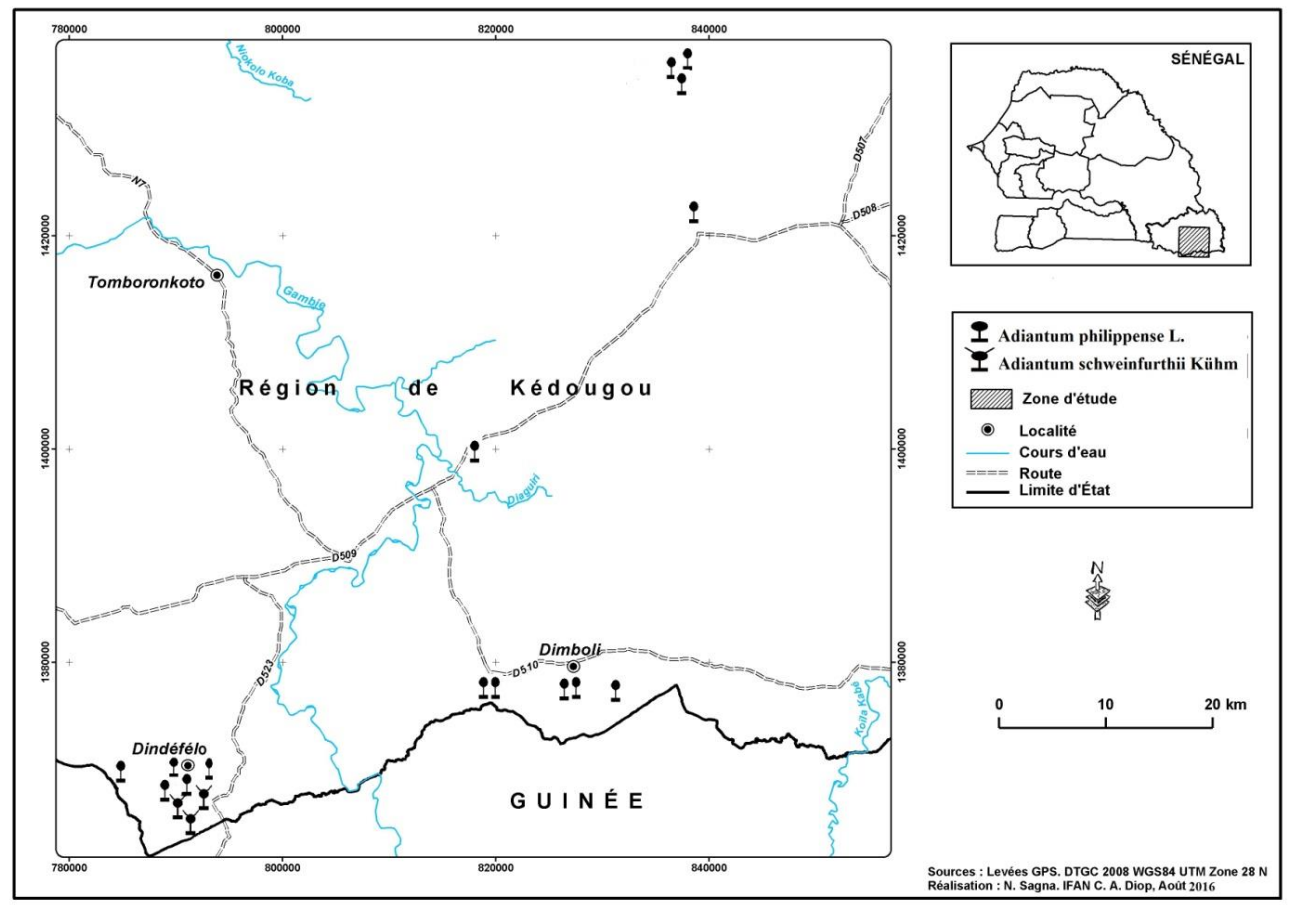

Figure 3. Carte de distribution des espèces du genre Adiantum dans les endroits humides de la région de Kédougou (Sénégal)

Au niveau de la cascade de Toumania, seules des Adiantum philippense L. ont été observées (Figure 3). La richesse des ptéridophytes au niveau des cascades est considérable avec une prédominance de la famille des Pteridaceae. Cependant, les genres Adiantum, Cyclosorus et Bolbitis sont les genres les plus représentatifs (Figures 3 et 4). Ainsi, au rang d'espèce, nous notons une large distribution d'Adiantum philippense L. et d'Adiantum schweinfurthii Kuhn. Elles ont été trouvées dans presque tous les sites prospectés mais, elles sont plus abondantes au niveau des cascades de Dindéfélo et de Dimboli (Figure 3). Cyclosorus dentatus (Forssk.) Ching., une Thelypteridaceae, a aussi été trouvée en abondance à Dindéfélo (Figure 4). Une certaine abondance relative de l'ensemble des espèces a été notée dans les environs des cascades de Dindéfélo et de Dimboli notamment au niveau des pistes qui y conduisent (Figures 3 et 4 ).

Certaines ptéridophytes sont plutôt rares et n'ont été trouvés que dans un seul ou au plus deux endroits. C'est le cas de Pteris sp., qui n'a été récoltée qu'au niveau de la cascade de Dindéfélo (Figure 4). S'agirait-il de 
l'espèce Pteris linearis ? Espèce signalée par Berhault en 1967 dans son ouvrage «Flore du Sénégal» sans que sa présence ne soit attestée par des spécimens dans les herbiers visités dans le cadre de cette étude. Cependant, un spécimen identifié comme Pteris linearis Poir. se trouve à l'herbier Dakar. Ce spécimen stérile récolté à Dindéfélo par Laweson et al. sous le numéro 7324 en 1990, ne permet pas de confirmer qu'il s'agit bel et bien de Pteris linearis Poir. Il en est de même pour les échantillons stériles identiques que nous avons récoltés durant notre prospection à Dindéfélo. En plus, la mission participative des herbonautes a signalé la présence de Pteris quadriaurita Retz., conservée à l'herbier de Paris $(\mathrm{P})$. Celle-ci a été récoltée par Heudelot sous le numéro 903 au Sénégal, sans aucune précision sur la date et la localité.

Le Sénégal, compterait-il deux espèces de Pteris ? Ou s'agirait-il juste d'une extrapolation de leur aire de distribution comme c'est plus souvent le cas.

Pellaea doniana J. Sm. ex Hook. et Nephrolepis bisserata (Sw.) Schott ont été récoltées uniquement à Dindéfélo (cascades de Dindéfélo et Afia2) et, Marsilea sp., exclusivement à Diaguiri (Figure 4).

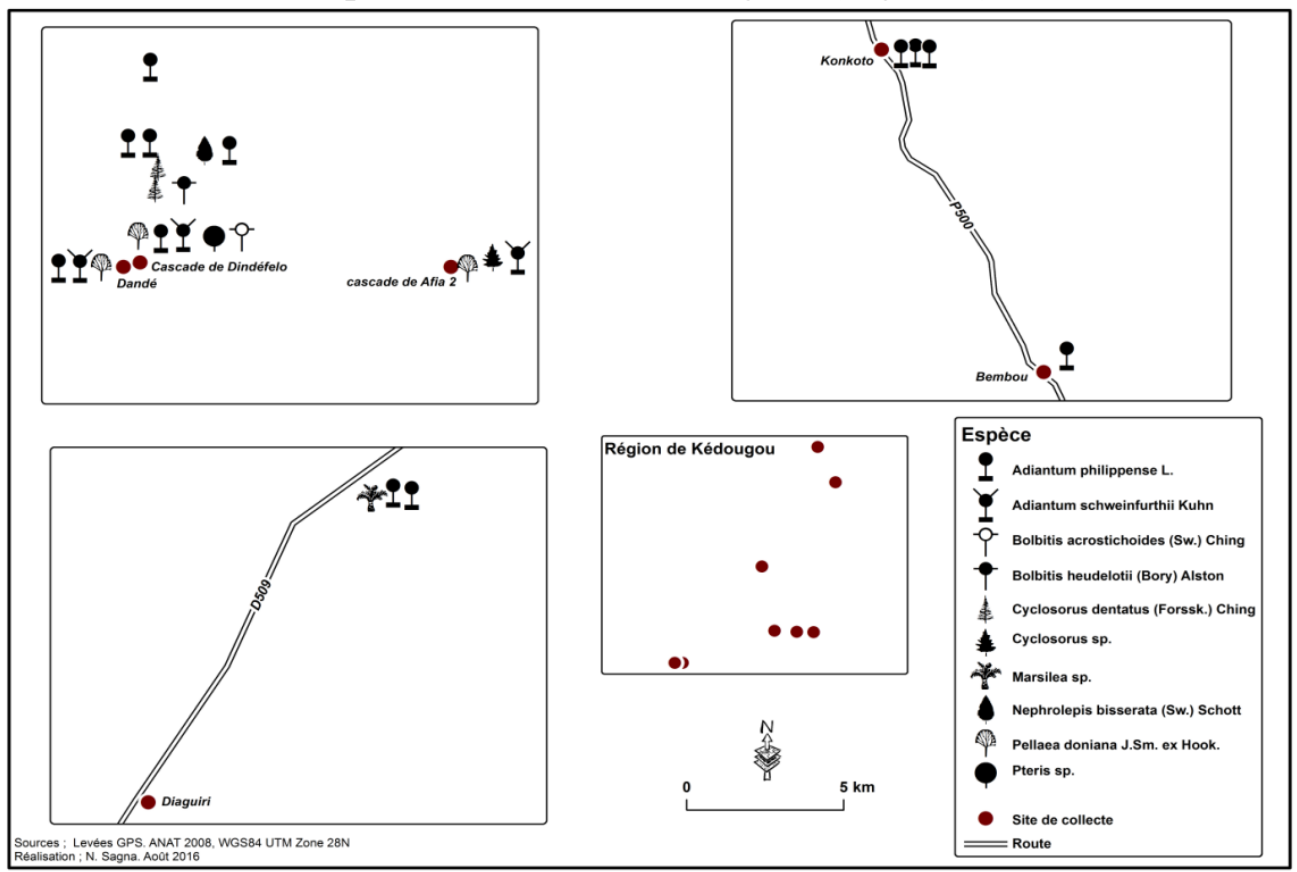

Figure 4. Carte de distribution des Ptéridophytes dans les endroits humides de la région de Kédougou (Sénégal)

\section{Discussion}

La comparaison de nos résultats à ceux de Bâ et Noba (2001) en ce qui concerne la flore générale du Sénégal, montre une presque similitude 
quant à la représentativité des familles des Ptéridophytes. Cependant, ces auteurs n'apportent aucune description détaillée de l'ensemble des familles ni des genres et des espèces. Le contenu de leur article se limite simplement à souligner l'importance des familles Marsileaceae, Thelypteridaceae et Adiantaceae face aux autres familles. Cette dernière famille, Adiantaceae, suite aux récentes révisions taxonomiques (Smith et al., 2006 ; Schuettpelz et al., 2007 ; Christenhusz et al., 2011) est placée dans la famille des Pteridaceae. En outre, il a été mis en relief d'une grande représentativité des genres Marsilea et Cyclosorus.

Il apparaît que les ptéridophytes sont plus représentés et diversifiés dans des zones humides voire zones de refuges avec cependant des adaptations dans des milieux ouverts notamment sur les pistes qui mènent aux cascades. La faible diversification des espèces dans certaines zones est due à l'interaction de divers facteurs écologiques, notamment l'anthropisation des forêts, facteur essentiel dans la région (Goudiaby, 1996) mais aussi la pédologie. L'humidité est aussi un facteur bien connu pour la plupart des espèces de ptéridophytes (Pausas \& Sáez, 2000). Certains auteurs ont démontré l'existence d'une relation claire entre la richesse des espèces et les paramètres du terrain (Pausas, 1994 ; Pausas \& Carreras, 1995 ; Pausas \& Sáez, 2000). En effet, l'étude faite dans le Nord-Est de la Péninsule Ibérienne par Pausas et Sáez (2000) a montré une plus importante richesse des ptéridophytes sur du substrat siliceux que sur du substrat calcaire. Les formations de sol de la zone de Dindéfelo se sont établies suites à la fissuration et la fragmentation des grès quartzites et des dolérites (Bassot, 1960 ; Michel, 1973 ; Goudiaby, 1996). Ceci étaye-t-elle la plus grande abondance des ptéridophytes à Dindéfélo par rapport aux autres zones prospectées ? Ainsi, des modèles de distribution des ptéridophytes de la région mériteraient une attention particulière.

$\mathrm{Du}$ fait de leur écologie particulière, la richesse floristique des Ptéridophytes varie beaucoup selon les changements des conditions abiotiques des milieux, expliquant pourquoi certaines espèces sont considérées comme bio-indicatrices (Kiew \& Anthonysamy, 1987 ; Barthlott et $a l ., 2001$; Mangambu et $a l ., 2013 b$ et 2015). Les ptéridophytes occupent une diversité d'habitats dans la région de Kédougou. Certaines comme Pellaea doniana J.Sm. ex Hook. ont été trouvées fixées sur des rochers suintants dans des endroits ombragés et d'autres comme Marsilea sp., Bolbitis heudelotii (Bory) Alston sont uniquement récoltées dans des eaux ruisselantes, des mares ou des flaques d'eau. L'ensemble des espèces colonise les bords des ruisseaux, les ravins profonds, les dessous des rochers et surtout les cascades.

Une faiblesse voire une absence totale d'espèces épiphytes a été notée dans la région. Seule Adiantum philippense L. a été trouvée épiphyte 
sur un Ficus umbellata Vahl. Il faut rappeler que des espèces peuvent être à la fois épiphytes et terrestres mais, A. philippense L. a été plus souvent retrouvée terrestre. Cette absence ou cette rareté d'espèces épiphytes dans la zone peut être due à la faiblesse de l'altitude car, durant notre prospection la plus haute altitude $(407 \mathrm{~m})$ a été enregistrée à Dandé. En effet, la plupart des études sur les épiphytes vasculaires de la région néotropicale ont confirmé que les habitats de montagne étaient plus favorables aux épiphytes vasculaires que les habitats de plaine (Nider et al., 1999). Toutefois, Johansson (1974) a démontré le contraire en mettant en évidence l'importance écologique des forêts de plaine. Il avait conclu dans ses études dans la région du Mont Nimba au Libéria, que la diversité des espèces épiphytes était plus importante entre 500-700 m au dessus du niveau de la mer qu'entre 1000-1300 m.

Cette première étude réalisée spécifiquement sur l'inventaire des ptéridophytes de quelques endroits humides de la région de Kédougou, présage des études approfondies sur ce groupe pour en assurer une meilleure connaissance et la conservation de certaines espèces sensibles qui seraient menacées de disparition.

\section{Conclusion}

Il ressort de cette étude que la flore ptéridologique des endroits humides de la région de Kédougou, est riche de 11 espèces, réparties dans 8 genres appartenant à 5 familles. Les Pteridaceae sont les plus représentatives dont l'espèce Adiantum philippense L. est largement distribuée dans la région.

La région apparaît pour beaucoup d'espèces comme faisant plus partie d'une limite d'extension que d'une véritable limite de répartition. En effet, les espèces se retrouvent visiblement nettement plus diversifiées en allant vers Dindéfélo.

La plupart de ces plantes préfère un climat ou une ambiance humide en sous bois, d'autres ont un besoin de rocailles. La région, riche en forêts galeries, et en cascades pourrait disposer d'une plus grande diversité, mais, son altitude inférieure à $600 \mathrm{~m}$ ne serait pas propice au développement d'espèces montagnardes. La déforestation, les activités minières, le tourisme en passant par les changements climatiques, constituent de sérieuses menaces pour ces endroits humides, habitats préférés des ptéridophytes. L'effet du tourisme se fait ressentir sur les ptéridophytes du fait que certaines espèces jalonnent les pistes qui mènent vers les cascades et ces espèces sont souvent piétinées par les randonneurs.

La connaissance locale des ptéridophytes est encore à améliorer. Ainsi, cette étude doit être étendue à la partie sud du Sénégal beaucoup plus humide pour une meilleure connaissance de la richesse de la flore 
ptéridologique. Une révision et une étude phylogénétique de ces espèces à l'échelle du Sénégal permettrait de mieux élucider la question. Cela doit être accompagné d'une meilleure gestion de cette flore par l'élaboration de plans de gestion et de conservation participatifs pour une utilisation durable de la diversité biologique. La conservation ex-situ des ptéridophytes dans les jardins botaniques et maisons peut être également envisagée.

Remerciements: Nous remercions sincérement Dr. G. Rouhan pour nous avoir aidé dans l'identification des espèces, et remercions également A.A. Camara curateur de l'herbier de Dakar. Nous remercions également S. Diop technicien au laboratoire botanique de l'IFAN et N. Sagna cartographe à l'IFAN.

\section{References:}

1. Aldasoro J. J., Cabezas F. \& Aedo C. 2004. Diversity and distribution of ferns in sub-Saharan Africa, Madagascar and some islands of the South Atlantic. Journal of Biogeography 31: 1579-1604.

2. Ba A.T. \& Noba K. 2001. Flore et Biodiversité végétale au Sénégal. Sécheresse 12 (3): 149-55.

3. Barthlott, W., Schmit-Neuerburg, V., Nieder, J. \& Engwald, S. 2001. Diversity and abundance of vascular epiphytes: a comparison of secondary vegetation and primary montane rain forest in the Venezuelan Andes. Plant Ecology 152: 145-156.

4. Bassot, J. P. 1960. Rapport fin de campagne 1958-1959. Bureau de Recherches Géologiques et Minières Dakar

5. Berhaut J. 1967. Flore du Sénégal. $2^{\mathrm{e} e}$ édition plus complète, avec les Forêts Humides de la Casamance. Dakar : édition Clairafrique ; 485 p.

6. Christenhusz M.J.M., Zhang X. \& Schneider H. 2011. A linear sequence of extant lycophytes and ferns. Phytotaxa 19: 7-54.

7. David R.G. 1993. Changing Aspects of Endemism and Endangerment in Pteridophyta. Journal of Biogeography 20: 293302.

8. Goudiaby A. 1996. Etude de la flore et de la végétation du site de Dindéféllo (Sud-est du Sénégal) éléments pour un aménagement. Thèse de doctorat, Université Cheikh Anta Diop de Dakar, 75p.

9. Johansson D. 1974. Ecology of vascular epiphytes in West African rain forests. Acta Phytogeographica Suecica 59: 1-129.

10. Johns R.J. 1985. Altitudinal zonation of pteridophytes in Papuasia. Proc. Roy. Soc. Edin. 86B: 381-389. 
11. Kiew, R. \& Anthonysamy, S. (1987). A comparative study of vascular epiphytes in three epiphyte-rich habitats at Ulu Endau, Johore, Malaysia. Malaysian Nature Journal, 41: 303-315.

12. Mangambu, M., Van Diggelen R., Mwangamwanga, J-C, Ntahobavuka,H. \& Robbrecht, E. 2013b. Espèces nouvellement signalées pour la flore ptéridologique de la République Démocratique du Congo. Int. J. Biol. Chem. Sci., 7(1) : 107-124.

13. Mangambu, M., Muhashy, F., Robbrecht, E., Janssen, T., Ntahobavuka Habimana, H., et Van Diggelenruurd. 2015. Ptéridophytes : Bio indicateurs des changements opérés sur la structure des forêts de zone de montagnes du Parc National de Kahuzi-Biega à 1'Est de la R.D. Congo. International Journal of Innovation and Scientific Research, ISSN 2351-8014 Vol. 16 No. 2 Jul. 2015, pp. 350-370.

14. Michel, P. 1973. Les bassins des fleuves Sénégal et Gambie. Etude géomorphologique. Thèse de Doctorat d'Ètat.Mém. ORSTOM 1973

15. Moran R. C. 2008. Diversity, biogeography, and floristics. Biology and Evolution of Ferns and Lycophytes, Ranker TA, Haufler CH, eds. Cambridge: Cambridge University Press. pp 367-394.

16. Nieder J., Engwald S., Barthlott W. 1999. Patterns of neotropical epiphyte diversity. Selbyana 20(1): 66-75.

17. Parris B.S. 1985. Ecological aspects of distribution and speciation in Old World tropical ferns. Proc. Roy. Soc. Edin. 86B: 341-346.

18. Pausas, J. G. 1994. Species richness patterns in the understorey of Pyrenean Pinus sylvestris forest. J. Veg. Sci. 5: 517-524.

19. Pausas, J. G. \& Carreras, J. 1995. The effect of bedrock type, temperature and moisture on species richness of Pyrenean Scots pine (Pinus sylvestris L.) forests. Vegetation 116: 85-92.

20. Pausas, J. \& Sáez, L. 2000. Pteridophyte richness in the NE Iberian Peninsula: biogeographic patterns. J. Plant. Ecol, 148, 195-205.

21. Pryer K.M., Schuettpelz E., Wolf P.G., Schneider H., Smith A.R. \& Cranfill R. 2004. Phylogeny and evolution of ferns (monilophytes) with a focus on the early leptosporangiate divergences. American Journal of Botany 91: 1582-1598.

22. Pryer K.M., Schneider H., Smith A.R., Cranfill R., Wolf P.G., Hunt J.S. \& Sipes S.D. 2001. Horsetails and ferns are a monophyletic group and the closest living relatives to seed plants. Nature 409: 618622.

23. République du Sénégal, 2014. Cinquième rapport national sur la mise œuvre de la Convention Internationale sur la Diversité Biologique, MEPN, 105 p. 
24. Roux J.P. 2003. Swaziland ferns and fern allies. Southern African Botanical Diversity Network Report No. 19. SABONET, Pretoria.

25. Roux J.P. 2001. Conspectus of Southern African Ferns. Southern African Botanical Diversity Network Report No. 13.

26. Schuettpelz E., Schneider H., Huiet L., Windham M.D. \& Pryer K.M. 2007. A molecular phylogeny of the fern family Pteridaceae: Assessing overall relationships and the affinities of previously unsampled genera. Molecular Phylogeny and Evolution 44: 11721185.

27. Smith A.R., Pryer K.M., Schuettpelz E., Korall P., Schneider H. \& Wolf P.G. 2006a. A classification for extant ferns. Taxon 55: 705731.

28. Tardieu-Blot M.-L. 1964. Ptéridophytes. In: Aubréville, Flore du Cameroun, 3. Paris.

29. Tardieu-Blot M.-L. 1953. Les ptéridophytes de l'Afrique intertropicale française. Mémoires de l'Institut Français d'Afrique Noire 28, 167-199.

30. Tryon R.M. 1985. Fern speciation and biogeography. Proc. Roy. Soc. Edin. 86B: 353-360. 